\title{
Development of Modified Medium for the Enhancement in Antifungal Activity of $P$. steckii (MF1 Mangrove Fungi) Against Verticillium Wilt Pathogenic fungi of Rose
}

\author{
Jyotasanmayee Sabat and Nibha Gupta* \\ Microbiology Laboratory; Department of Biotechnology; Regional Plant Resource Centre; Bhubaneswar - 751015
} (Orissa) - India

\begin{abstract}
The antifungal activity of Penicillium steckii (MF1) against pathogenic fungi of rose causing verticillium wilt was evaluated under differential nutrient condition. A suitable and modified medium was obtained in which test fungus exhibited enhanced antifungal activity and produced larger inhibition zone against the verticillium fungus. The study carried out to modify the medium components at level of nitrogen, carbon source and their concentration on agar plates. Addition of amino acids, metals and plant growth hormones did not show positive effect but glycerol enhanced the antifungal activity very much.
\end{abstract}

Key words: antifungal, Penicillium, nutrient, rose, Verticillium wilt

\section{INTRODUCTION}

The plants suffer from diseases by both agents of abiotic and biotic nature. The biotic causes are mainly pathogenic microorganisms. The fungi, bacteria and mycoplasma are organizational entities of heterotrophic nature and distinguished as parasites and pathogens. The rose is a popular garden plant and important commercial cut flower grown both in the open field as well as under glass house conditions but fungal diseases often limit the productivity as well as the commercial values. The most common diseases that affect roses are caused by fungi i.e. Powdry mildew, Black spot, Rust, Verticillium wilt, Downy mildew, Brown canker, Black mold, Botrytis blight, Dieback, etc. (Horst, 1983). Though several fungal diseases in rose have been reported ( Morandi et al., 2001; Kaminska et al., 2001; Xu, 1999 and Uchneat et al., 1999) but their occurrence as well as importance of many of the causal fungi are yet to be investigated.

Several reports are available on antifungal activity of fungi as well as Penicillia (Soman et al., 1999 Garcialkirchner et al., 2000, Klich et al, 1998, Engstrom et al.,, 1999, Someya et al. 2000; Benhamou and Brodeur, 2000; Vangulik et al., 2001). Such studies are gaining much importance due to source of fungi i.e. mangrove ecosystem and secondly due to their effectiveness towards the rose pathogen, especially verticillium wilt fungi. In the present study, basal PDA medium was modified to obtain better antifungal activity by using different $\mathrm{C}$ and $\mathrm{N}$ source and other growth stimulating factors in the laboratory conditions.

\footnotetext{
* Autor for correspondence: nguc2003@yahoo.co.in
} 


\section{MATEIRALS AND METHODS}

\section{Source of fungi}

The verticillium wilt pathogenic fungi was isolated from the infected portion of rose plants, purified and identified morphologically (Mehrotra and Aneja, 1990; Barnett and Hunter, 1972; Kenneth and Horst, 1983).The fungi tested for antifungal activity against rose pathogenic verticillium fungi was obtained from culture collection of Microbiology Laboratory, Regional Plant Resource Centre, Bhubaneswar that was isolated from mangrove ecosystem of Bhitarkanika, Orissa.

\section{Analysis of antifungal properties}

All the test organisms were tested for their antimicrobial activity against four rose pathogenic fungi by inoculating them through co-inoculation method on potato dextrose agar medium and kept incubation at $30^{\circ} \mathrm{C}$ for 5- 7 days. The inhibition zone around the colony of potent fungi were measured with the help of Hiantibiotic zone scale. Antifungal activity was determined by zone of inhibition ( $\mathrm{mm}$ ). Relative magnitude of inhibition was calculated by following formulae (Darokar et al.1999; Sing et al,. 1999)

Relative magnitude of Inhibition $=$ Area defined by zone of Inhibition/ Area defined by the disc.

Percent Inhibition $=$ Colony diameter in check Colony diameter in treatment/ Colony diameter in check x 100 .

\section{Effect of Nutritional amendments}

During the screening program, the potato dextrose medium was found to be the best medium for higher antimicrobial activity. Hence, the potato dextrose agar medium was taken as the basal medium for the modification of nutritional amendments.

\section{Effect of potato extract}

The different concentration of potato extract taken was 5,10,15,20,30, 50, 100 and $150 \%$ in place of $25 \%$ of basal potato dextrose medium.

\section{Effect of dextrose concentration}

The concentration of dextrose taken were 0.5 , $1.0,1.5,2.5$ and $3.0 \%$ in place of $2 \%$ taken in control set.

\section{Effect of other carbon sources}

The different carbon sources taken were sucrose, lactose, starch, and manitol. In each case the carbon source was taken at $2 \% \mathrm{w} / \mathrm{v}$ and added to the basal PDA medium in place of dextrose.

\section{Effect of combination of carbon sources}

In combination with dextrose other carbon sources were taken, viz., sucrose, lactose, starch, manitol at the rate of $2 \%$ and added to the basal medium.

\section{Effect of concentration of selected carbon source}

Sucrose and lactose were found as the best carbon source for this fungal activity against dieback and verticillium pathogenic fungi, respectively. The following concentrations $0.5,1.0,1.5,2.5$ and $3.0 \%$ $(\mathrm{w} / \mathrm{v})$ were taken in a higher order to determine its optimum activity.

\section{Effect of selected carbon source and glycerol}

In combination with sucrose at different concentration, $1 \%$ glycerol was added to the medium and observed for antifungal activity under co inoculation method.

\section{Effect of concentration of glycerol}

Sucrose $(2.5 \% \mathrm{w} / \mathrm{v})$ was found to be most suitable. With this concentration of sucrose different $\%$ of glycerol was taken i.e. 0.5,1.0, 1.5, 2.0, 2.5, $3.0 \%$.

\section{Effect of amino acids}

The different amino acids taken $(0.1 \%$ w/v) were Larginine, L-glutamic acid, L-methionine, L-tyrosine, L- valine, L- aspargine, lysine, alanine, proline, threonine, phenylalanine, glutamine and tryptophan .

\section{Effect of concentration of amino acids}

The amino acid with which organism showed best activity, were taken and added to the medium at $0.05,0.1,0.5,1.0,1.5$, and $2.0 \%(\mathrm{w} / \mathrm{v})$.

\section{Effect of potassium salts}

Two potassium salts i. e. $\mathrm{KCl}$ and $\mathrm{KH}_{2} \mathrm{PO}_{4}$ were added to the modified medium at the rate of $0.5 \%$.

\section{Effect of Ammonium and sodium salts}

Along with the selected carbon sources in combination, glycerol (\%) and amino acid (\%), medium was added with different ammonium salts at the rate of $1 \%$. These were ammonium chloride, ammonium ferrous sulphate, ammonium fluoride, 
ammonium sulphate, ammonium metavendate, ammonium molybdate, ammonium dihydrogen orthrophosphate, ammonium nitrate, ammonium oxalate, ammonium acetate, sodium sulphate and sodium thiosulphate.

Effect of heavy metals and their concentration

To determine the effect of metal ions, $\mathrm{MgCl}_{2}$ and $\mathrm{MnSO}_{4}$ were taken in different concentration i.e. $0.01,0.03,0.05,0.07$ and 0.1 $\%$ into the modified medium.

\section{Effects of hormones and their concentration}

To determine the effect of the plant growth hormones, naphthyl acetic acid, benzyl adenine, Kinetin, and gibberalic acid were taken in concentration of 10, 15, 20 and $25 \mathrm{ppm}$.

\section{RESULTS AND DISCUSSION}

\section{The Effect of potato extract}

The media taken was basal type, i.e. potato dextrose agar. The data (Fig. 1) showed that the organism preferred $25 \%$ potato extract for giving higher zone of inhibition to the other concentration of potato extract taken. The inhibition zone and RMI recorded was $7 \mathrm{~mm}$ and 2.8, respectively (Fig. 1). In this experiment, $25 \%$ potato extract showed $42.8 \%$ inhibition that was much higher as compared to other concentrations of potato extract taken (except one where $50 \%$ concentration of potato extract was used and $43.3 \%$ inhibition was obtained).

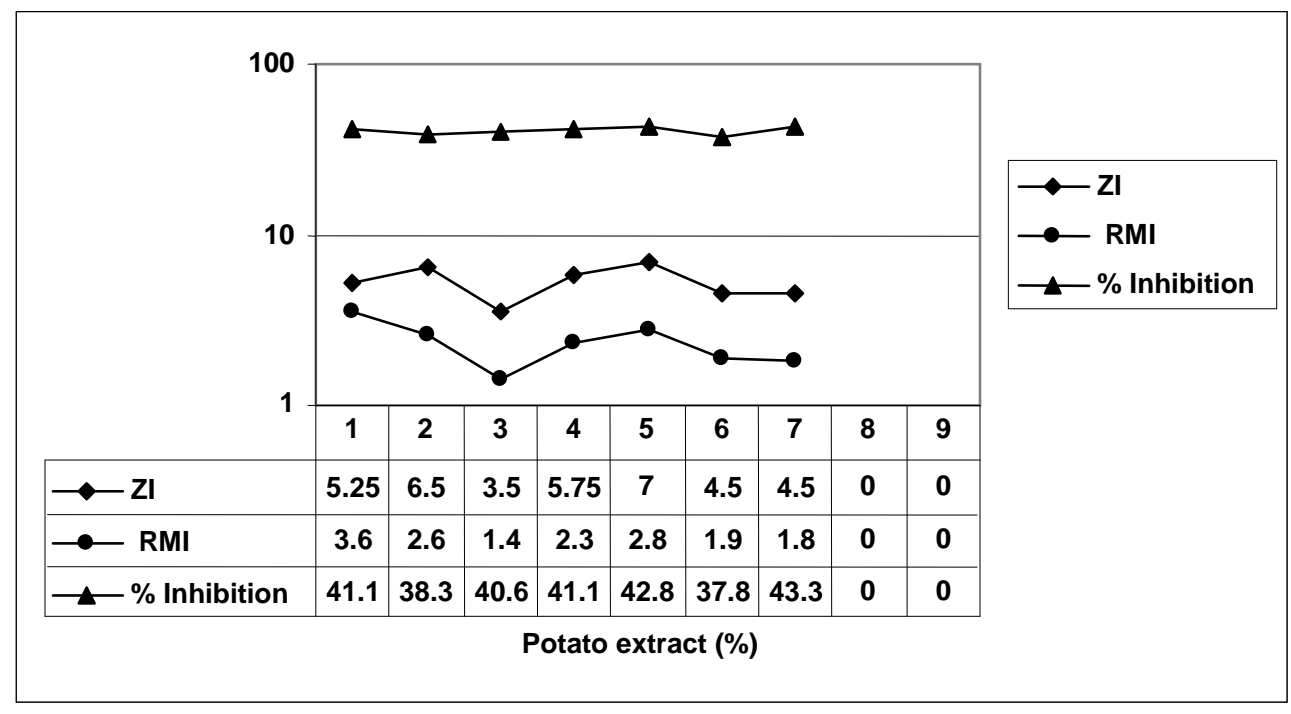

Figure 1 - Effect of potato concentration Dextrose $(2 \%)+$ Potato extract $[1=5 \%, 2=10 \%$, $3=15 \%, 4=20 \%, 5=25 \%, 6=30 \%, 7=50 \%, 7=50 \%, 8=100 \%, 9=150 \%]$.

\section{The Effect of dextrose concentration}

Out of six different concentration of dextrose tested, the highest zone of inhibition was observed with $2 \%$ dextrose i.e. $6.5 \mathrm{~mm}$ (Fig. 2). At other concentrations of dextrose, the fungus did not produce good inhibition zone. Therefore, $2 \%$ dextrose was selected for further studies. Relative magnitude of inhibition was higher at this concentration of dextrose, i.e. 2.6 where as higher value of $\%$ inhibition was observed in experimental set up in which dextrose was used at 2.5 and $3.0 \%$ concentration.

\section{Effect of other carbon sources}

Out of four carbons sources taken, viz. lactose, sucrose, starch and manitol, the best result obtained with sucrose (Fig. 3). In PDA containing $25 \%$ potato extract, $2 \%$ sucrose exhibited $4.5 \mathrm{~mm}$ zone of inhibition. This also showed highest RMI i. e. 1.8 and $38.33 \%$ inhibition, which was quite similar with the other carbon sources used. 


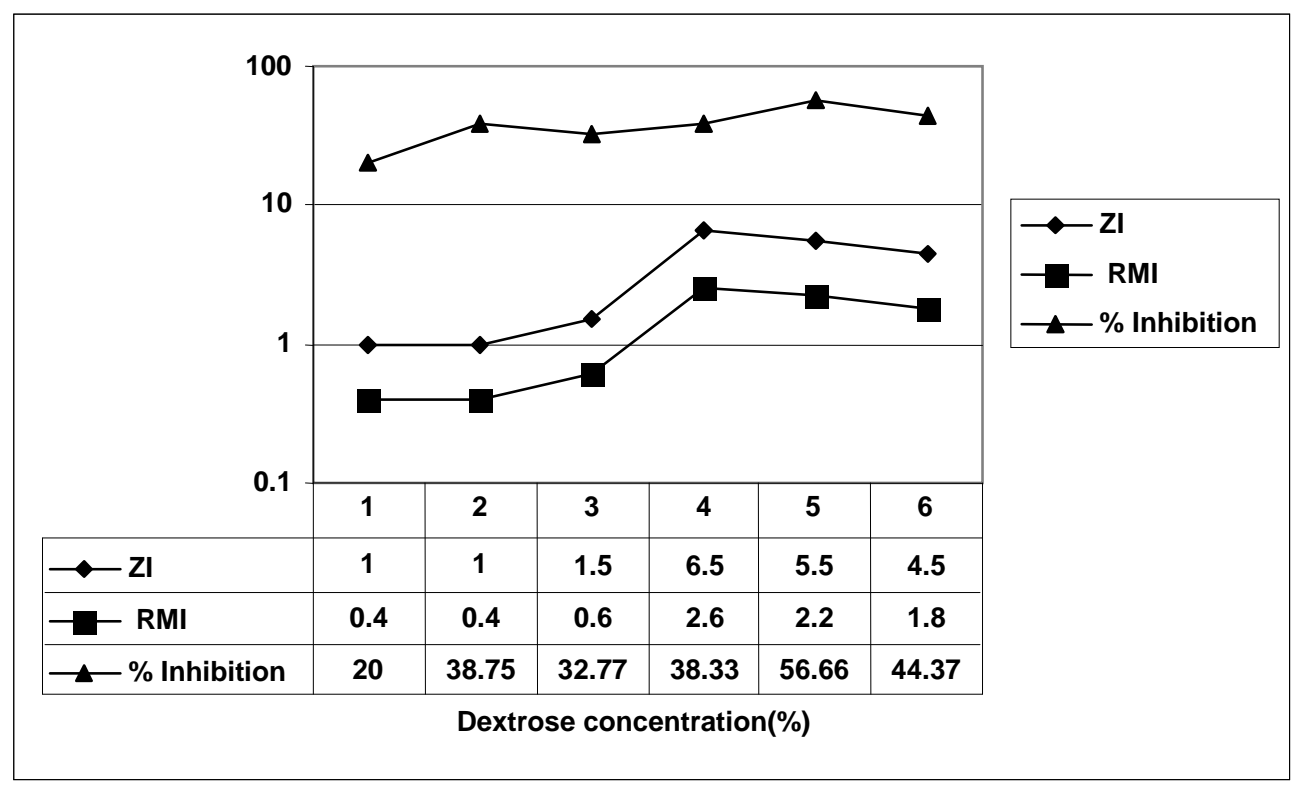

Figure 2 - Effect of Dextrose Potato extract $(25 \%)+$ Dextrose concentration [ $1=0.5 \%, 2=1.0 \%$, $3=1.5 \%, 4=2.0 \%, 5=2.5 \%, 6=3.0 \%]$.

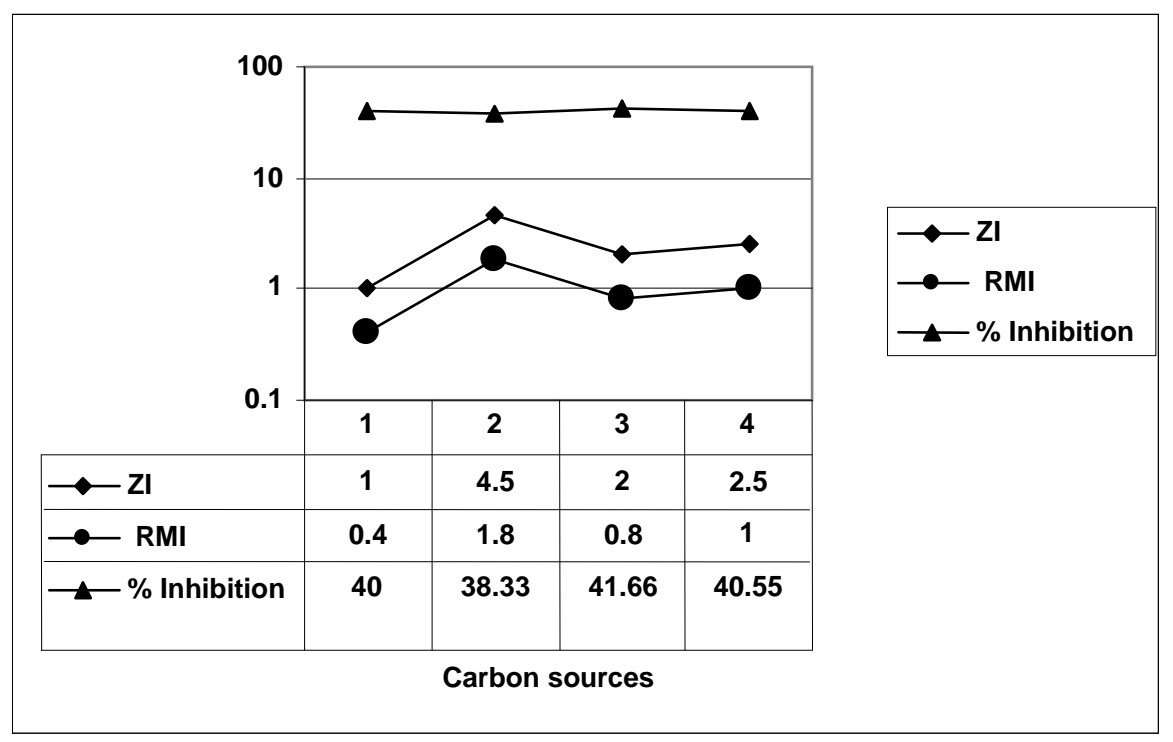

Figure 3 - Effect of carbon sources Potato extract $(25 \%)+$ Other carbon sources (2\%), 1= Lactose, $2=$ Sucrose, 3 = Starch, 4 = Manitol.

Effect of other carbon sources along with dextrose

Four carbons sources i.e. lactose, sucrose, starch and manitol were taken separately in combination with $2 \%$ dextrose $+25 \%$ potato extract. These carbon sources exhibited good inhibition against the test pathogen than the only carbon source used (Fig. 4). Lactose exhibited highest zone of inhibition, RMI and \% inhibition but comparatively it was lower than the dextrose used alone. Hence, the different concentration of lactose was taken along with dextrose $(2 \%)$. 


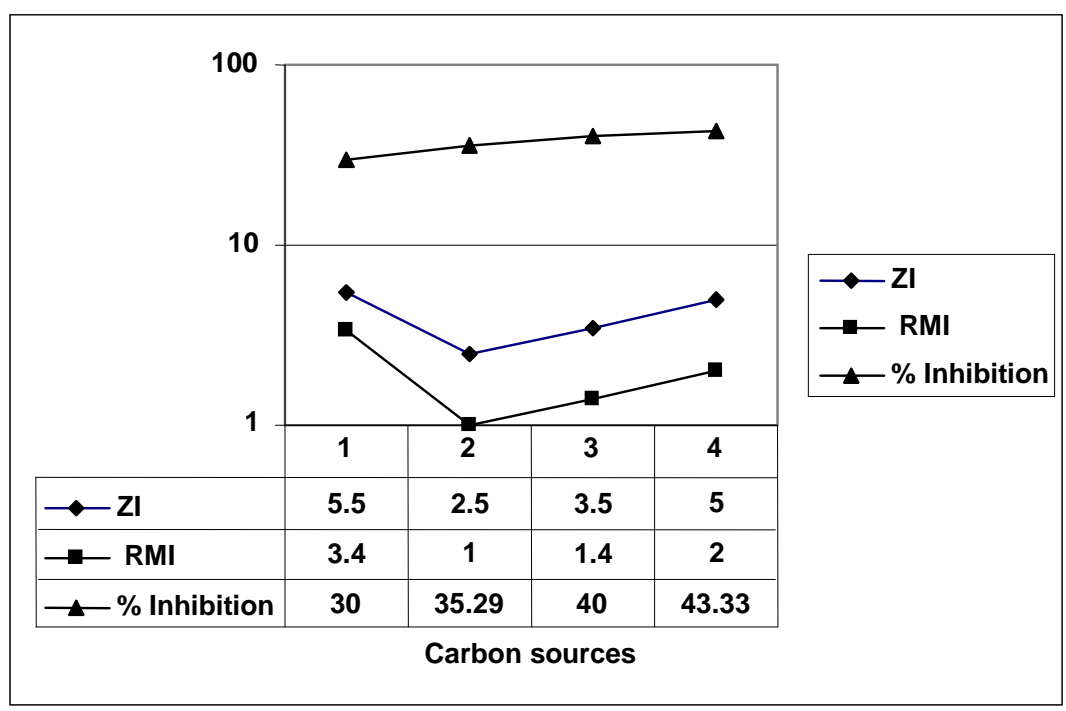

Figure 4 - Effect of dextrose and other carbon sources Potato extract $(25 \%)+$ Glucose $(2 \%)+$ Other carbon sources $(2 \%) 1=$ Lactose, $2=$ Sucrose, $3=$ Starch, $4=$ Manitol.

\section{Effect of concentration of Lactose:}

Lactose was taken at different concentration along with $25 \%$ potato extract and $2 \%$ dextrose (Fig. 5). Concentration wise, the best concentration $1 \%$ exhibited $7.5 \mathrm{~mm}$ zone of inhibition, which was highest among the all previous experimental sets taken. The other concentration of lactose did not show good inhibition zone and RMI. However, at $1 \%$ concentration of lactose in combination with $2 \%$ dextrose, the highest level of inhibition on growth of the pathogenic fungus was observed. Therefore, along with $25 \%$ potato extract lactose $(1 \%)+$ dextrose $(2 \%)$ was taken as carbon source.

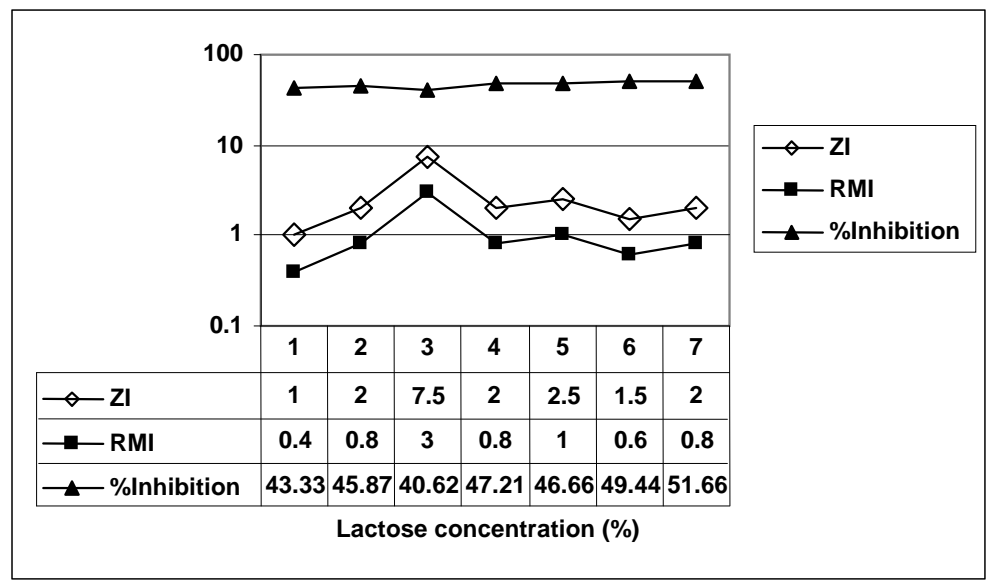

Figure 5 - Effect of lactose concentration Potato extract $(25 \%)+$ Glucose $(2 \%)+$ lactose $1=0.25 \%, 2=0.50 \%, 3=1.0 \%, 4=1.5 \%, 5=2.0 \%, 6=2.5 \%, 7=3.0 \%$. 


\section{Effect of glycerol}

Glycerol was taken at the rate of $1 \%$ into the modified medium along with lactose and dextrose (Fig. 6). The addition of glycerol showed positive effect and gave $11.5 \mathrm{~mm}$ of inhibition zone at $1 \%$ concentration level.
Results exhibited the positive effect of combination of lactose, dextrose and glycerol. The enhancement in the RMI i.e. 4.6 also suggested the usefulness of glycerol in combination with lactose and dextrose for the further modification of medium.

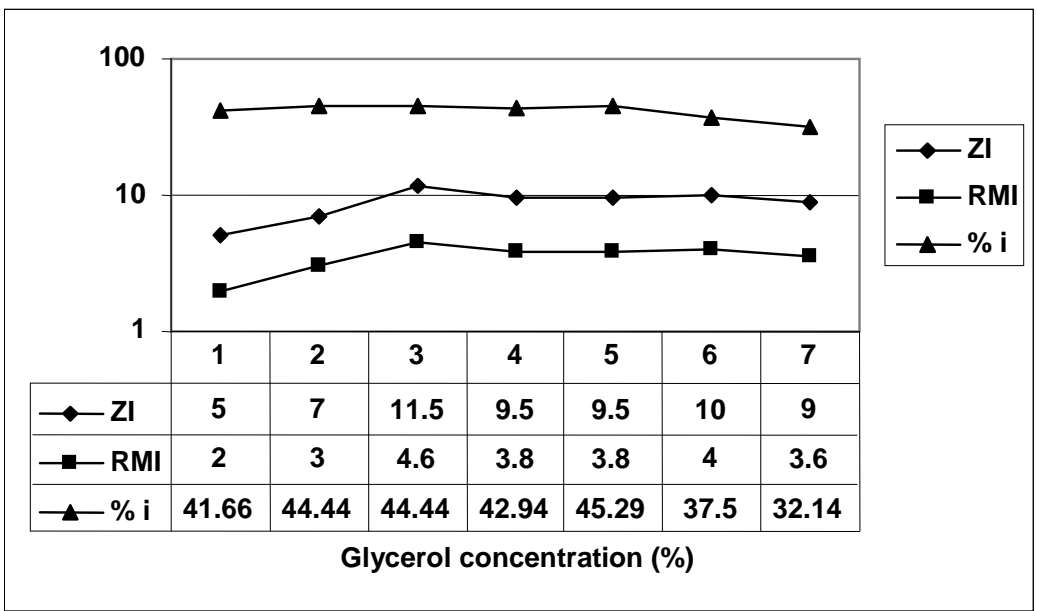

Figure 6 - Effect of glycerol Potato extract 25\% +glucose (2\%), Lactose (1\%), Glycerol (\%) :1=0, $2=0.5 \%, 3=1.0 \%, 4=1.5 \%, 5=2.0 \%, 6=2.5 \%, 7=3 \%$.

\section{Effect of amino acids}

Total 13 amino acids were tested for the further medication of medium at $0.1 \%$ concentration (Fig. 7). In case of all amino acids tested, the inhibition of growth of test pathogen could be observed but significant change in inhibition was observed with valine that exhibited $7.5 \mathrm{~mm}$ zone of inhibition. Though valine could not produce the inhibition zone as good as obtained with glycerol combination, its effects was evaluated by taking different concentration into the modified medium.

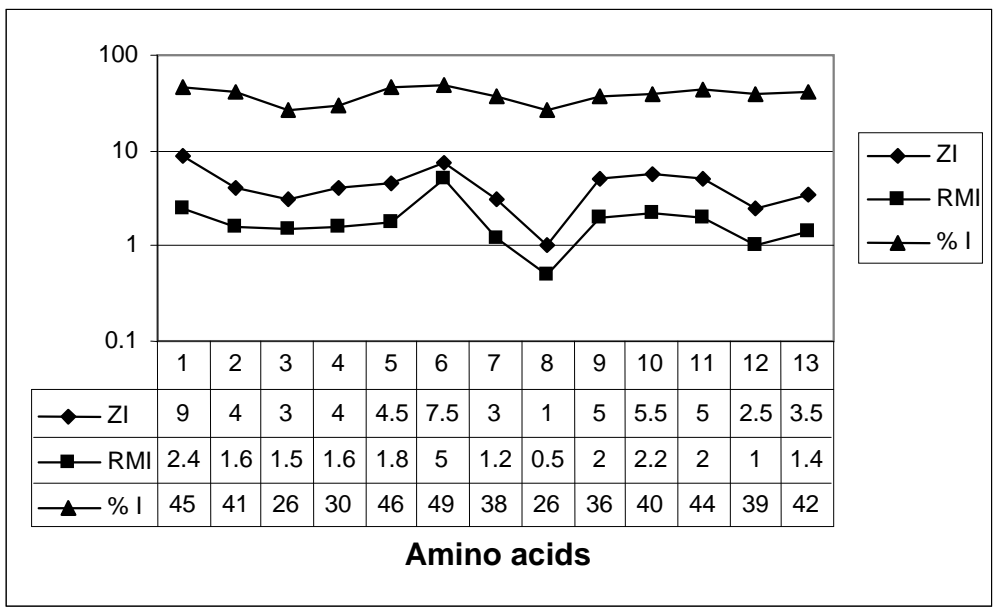

Figure 7 - Effect of aminoacids Potato extract 25\% +glucose (2\%), Lactose (1\%), Glycerol 1\%,and amino acids $1=$ no amino acid, $2=\mathrm{L}$ arginine, $3=\mathrm{L}$ Glutamic acid, $4=\mathrm{L}$ Methionine, $5=\mathrm{L}$ Tyrosine $6=\mathrm{L}$ valine, $7=\mathrm{L}$ asparagine, $8=$ Lysine, $9=$ alanine, $10=$ proline, $11=$ threonine $12=$ phenylalanine, $13=$ glutamine, $14=$ tryptophan . 


\section{Effect of concentration of Valine}

Six different concentration of valine were tested in combination with the modified medium (Fig. 8). The results obtained showed that $0.1 \%$ concentration exhibited good inhibition towards the growth and produced $8.05 \mathrm{~mm}$ zone around the growing colony of test pathogen. Valine in low concentration did not produce good results.

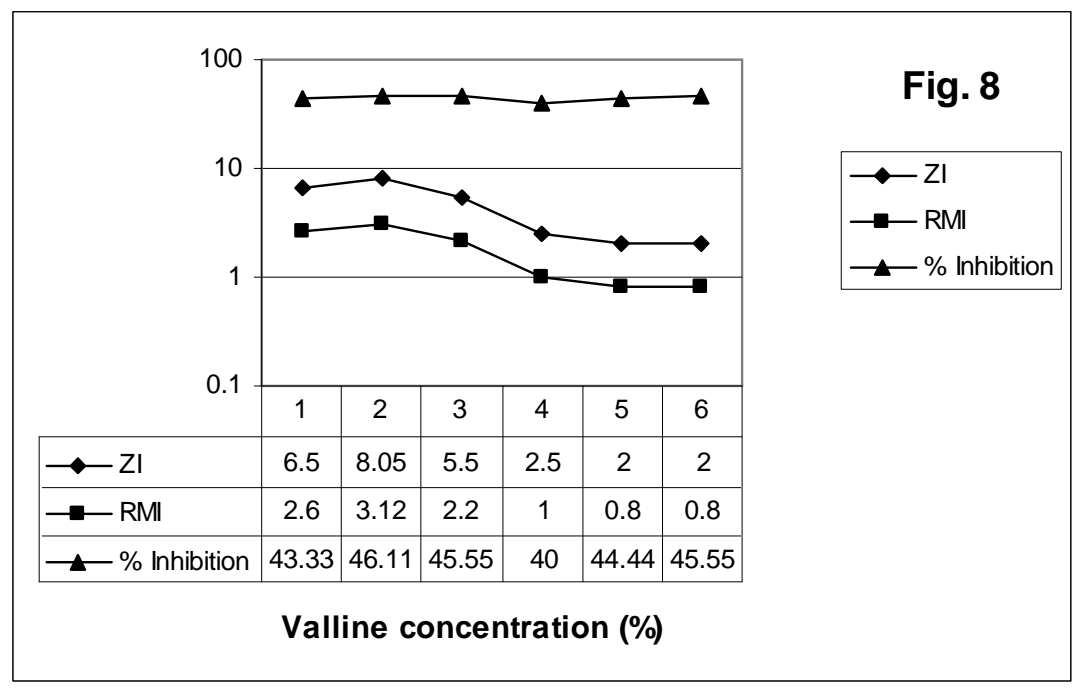

Figure 8 - Effect of valine Potato extract $25 \%$ +glucose (2\%), Lactose (1\%), Glycerol $1 \%$, and valine $1=0.05 \%, 2=0.1,3=0.5 \%, 4=1.5,5=2.0,6=2.5$.

\section{Effect of metals}

Metal salts used in this study were $\mathrm{MgCl}_{2}$ and $\mathrm{MnSO}_{4}$ in different concentration. The effect of both metal salt was negative upon the growth of pathogenic fungi (Verticillium). Therefore, no metal salt was selected further.

\section{Effect of Hormones}

Four plant growth-promoting hormones were tested into the modified medium at different concentration. There was a negative effect of all the four hormones on the growth of pathogenic fungi of verticillum. Therefore no plant growth hormone selected for the further modifications.

\section{Effect of ammonium, sodium and potassium salts}

There was no effect on antifungal activity of Penicillium steckii, rather the growth of the organism was retarded as compared to previous modification sets. Therefore, no salt were selected for the further modification of the medium.

\section{Development of modified medium}

Based on the results the medium contained $25 \%$ potato extract, $2 \%$ dextrose, $1 \%$ lactose, $1 \%$ glycerol, $\mathrm{pH} 5$ and incubation period 6 days in solid condition. In original and normal medium of PDA (pH 5.0), the fungi produced $7.0 \mathrm{~mm}$ zone of inhibition whereas in the modified medium it exhibited $11.5 \mathrm{~mm}$ zone of inhibition. The modified medium developed through this study showed the enhanced antifungal activity of fungus towards verticillium pathogenic fungi.

In the present study, Penicillium steckii (MF1) was selected because it was found to be active against verticillium wilt pathogenic fungi occurring frequently among the rose varieties. This fungus preferred potato dextrose agar medium and exhibited highest antifungal activity against this pathogenic fungi under laboratory conditions. Hence, PDA was selected for the modification.

It is very well known that natural media contain indefinite composition and some of them are used for the industrial production of antibiotics, amino acids, vitamins and other valuable products of metabolism of microorganims (Egorov, 1985). Hence, it was thought to modify this basal medium first to obtain best performance of Penicillium steckii (MF1) and modifications at the level of carbon, nitrogen, and growth stimulants, etc. 
The character of a given physiological and biochemical process occurring in microorganims can be elucidated only by cultivating them on thought selected media. Selection of suitable medium for cultivation of microorganism is very important to reveal their potential ability to produce antimicrobial compounds especially antibiotics. The experimental studies carried out to evaluate cultural requirement for P.steckii have provided good information on the effects of test parameters upon antifungal activity on solid state of the medium. Nutritional modifications such as carbon source, nitrogen sources etc. affected the antifungal activity of $P$. steckii.

The medium taken was basal type because in previous studies this organism had shown preference for the potato dextrose agar of $\mathrm{pH} 5$ with respect to the antifungal activity towards the verticillium pathogenic fungi. This was further corroborated with the facts reported by several workers who obtained PDA as the best medium for the growth of some fungi (Selvan and Setharaman, 2003; Leon et al, 1999; Aneja, 1993). Qualitative characteristics of the medium components are very important in the cultivation of organisms for the study of their antibiotic properties. The presence in the medium of different forms of carbon and /or nitrogen, and any other components induces different response in the microbes, depending on the presence of enzymatic and metabolic activity. The direction of the metabolic reactions thus can be varied. This may activate the antibiotic properties or, on the contrarily, inhibit formation of antibiotics. It has been reported that glucose containing media sometimes adversely affects the growth of organisms indirectly to metabolisms (Carter and Bull 1969, Siddiqui and Shaukat, 2002). The present fungus utilized lactose as the primary source of carbon. The effect of lactose was found to be suitable as a factor enhancing the antifungal activity of the test fungi (Simley et al. 1967; Varshney, 1981). The results showed the positive effect of lactose with combination of glucose in enhancing the antifungal activity against the verticillium wilt fungus. The combination of dextrose and lactose was found better than the dextrose used.

The presence of glycerol (alcohol) was also reported to be favorable on the growth of organism and yield of antibiotics in some organisms (Siddiqui and Shaukat, 2002; Ismet et al., 2004). The positive effect of glycerol was exhibited in present study for the enhancement of the antifungal activity tested against verticillium wilt pathogen. The addition of glycerol $(1 \%)$ enhanced the antifungal activity of the fungus.

The postassium salts had no effects on the fungal activities. Similarly, different salts of ammonium did not show any effect upon the antifungal activity of this fungus. Though several ammonium and potassium salts were reported to be effective in enhancing the growth and metabolism of the fungus (Sood, 1990). The fungus did not respond towards the utilization of potassium salts for its antifungal properties, rather it was poor in this condition. Ammonium salts are most readily assailable forms of nitrogen for many organisms but P. steckii (MF 1) did not exhibit any enhanced activity in the presence of ammonioum salts.

Amino acids are important for the metabolism of microbes and directly involved in the synthesis of proteins and take part in the formation of antibiotics. Amino acids were reported as good source of nitrogen and accelerated the biomass production of fungi. Among them alanine, glycine arginine and aspartic acid were the best source for the growth of bioagents (Pateman and Kinghorn, 1976; Monga , 2001). This was be true present study as the fungus did not exhibit good response in the presence of amino acid when tested against the verticillium wilt pathogenic fungus. Addition of valine could somehow be able to enhance the antifungal activity but not up to the level of earlier experiments.

Some growth factors that affect the metabolic efficiency of fungi are trace elements, vitamins and hormones. The plant growth hormones and metals salts are quite well known for the positive impact on physiology of certain microbes (Sood, 1990). Some such compound were evaluated for their effects on antifungal activity of Penicillium steckii. The negative effects of hormones towards the enhancement of antifungal properties of $P$. steckii were observed. The growth promoting and retarding effects of metal salts have also been reported. The effect of both the metal salts was negative on the antifungal activity. Thus it was not worthy of incorporation of metals and hormones into the medium.

The present study exhibited the achievement of suitable modified medium in which the antifungal activity by $P$. steckii could be obtained. This study was also suggested the good production of antimicrobial agent at laboratory level and potential of scaling up the process. The optimization of nutritional conditions needs to 
more standardize to be used as key factor in prerequisite industrial processes to achieve the product in desired amount.

\section{ACKNOWLEDGEMENTS}

NG is thankful to Ministry of Environment and Forests, Govt.of India for financial support through project No. 22/7/2003-CS /BR.

\section{REFERENCES}

Aneja, K.R. (1993), Experiments in microbiology plant pathology and tissue culture, Wishwa prakashan, Delhi, pp.117-147.

Barnett, J.L. and Hunter, B.B. (1972), illustrated genera of imperfect fungi. Burgess Publishing Company, Minneapolis, Minnesota, pp.88.

Benhamou, N. and Brodeur, J. (2000), Evidence for antibiosis and induced host defense reactions in the interaction between the Verticillium lecani and Penicillium digitatum, the causal agent of green mold. Phytopathology, 90(9): 932-943.

Carter, B.L.A and Bull, A.T. (1969), Studies of fungal growth and intermediary carbon metabolism under steady and non steady conditions. Biotechnology and Bioengineering. 11: 785-804.

Daroker, M.P.; Khanuja, S.P.S. ; Bagchi, G.D. and Kumar, S. (1999), Spectrum of antibacterial activity possessed by petals of rose varieties. Current Science, 77(10): 1238-1241.

Egorov, N.S. (1985), Antibiotics: A scientific approach. MIR publishers, Moscow.

Engstrom, K.; Widmark, A.K.; Brishammar, S. and Helmersson, S. (1999), Antifungal activity to phytophthora infestans of sesquiterpenoid from infected potato tubers . Potato Research, 42(1): 4350.

GarciaKirchner, O.; Segura Granados, M.; RobledoBautista, I. and Duranparamo, E. (2000), Screening of potential antibiotic action of cellulolytic fungi. Applied Biochemistry and Biotechnology, 84(6): 769-778.

Horst, R.Kenneth. (1983), Compendium of rose diseases published by the American Phytopathological Society in cooperation with Dept of plant pathology, Cornell Univ.

Ismat, A.; Vikineswary, S.; Paramaswari, S.; Wong, W.H.; Ward, A., Seki, T.; Fiedler, H.P. and Goodfellow, M. (2004), Production and chemical characterization of antfungal metabolites from micromonospora sp. M39 isolated from mangrove rhizosphere soil. World Journal of Microbiology and Biotechnology, 20(5): 523-528.
Kaminska, M.;Dziekanowska, D. and Rudzinskal, A. (2001), Detection of phytoplasma infection in rose, with degeneration symptoms. Journal of Phytopathology, 149(1): 3-10.

Mehrotra, R.S. and Aneja, K.R.(1990), An introduction to mycology. Wiley Eastern Limited, New Delhi, pp.582-583.

Monga, D.(2001), Effect of carbon and nitrogen sources on spore germination, biomass production and antifungal metabolites by species of Trichoderma and Gliocladium. Indian Phytopathology, 54(4): 435437.

Morandi, M.A.B.; Maffia, L.A. and Sutton, J.C. (2001), Development of Clonostachys rosea and interactions with Botrytis cinerea in rose leaves and residues. Phytoparasitica, 29(2): 103-113.

Pateman, J.A and Kinghorn, J.R. (1976), Nitrogen metabolism in the filamentous fungi. Volume II. Eds. J.E.Smith and D.R.Berry, Edward Arnold, London: 159-237.

Siddiqui, I.A. and Shaukat, S.S. (2002), Zinc and glycerol enhance the production of nematicidal compounds invitro and improve the biocontrol of Meloidogyne javanica in tomato by Fluorescent Psedomonads, Letters in Applied Microbiology, 35(3): 212-217.

Sing, Dipak.; Chandra, J.P. and Sing, A.B. (1999), Response of fungicides and antibiotics against Anthracnose of poplar caused by Colletotrichum graminicolum. Indian Forester, 125(6): 566-572.

Smiley, K.L.;M.C.Cadmus, Patricia, Liepins.(1967), Biosynthesis of D-manitol from D-glucose fron Aspergillus candidus. Biotechnol. Bioeng, 9(3): 365374.

Soman, A.G. ; Gioer, J.B. ; Koster, B. and Malloch, D. (1999), Sporovexins A-C and a new preussomerin analog : Antibacterial and antifungal metabolites from the coprophilous fungus Sporomiella vexans. Journal of Natural products, 62(4): 659-661.

Someya,N.; Nakajima, m.;Hirayae, K.; Hibi, T. and Akutsu, K. (2000), Biological control of cyclamen soil borne diseases by Serratia marcescens strain B2 . Plant Disease, 84(3):334-340.

Sood, M.(1990), Studies on Aspergillus umbrosus (Bainier and Sartory) as related with the physiology of growth and antibiotic production. Phd. Thesis Department of Bioscience, Ravisankar University, Raipur.

Suriachandraselvan, M and K. Seetharaman (2003), Effect of culture media on growth and sclerotial production of different isolates of Macrophomina phaseolina infecting sunflower. J. Mycol. Pl. Pathol., 33(2): 226-229.

Suriachandraselvan, Leon, B.M.; Argaiz, A. and Lopez, M.A.(1999), Individual and combined effect of vanillin and potassium sorbate on Penicillium digitatum, Penicillium glabrum and Penicillium 
italicum growth. Journal of Food Protection, 62(5):540-542.

Uchneat, M.S. ; Spicer, K. and Craig, R. (1999), Differential response to floral infection by Botrytis cinerea within the genus Pelargomium. Hortscience, 34(4): 718-720.

Van Gulik, W.M., M.R. Antoniewicz, W.T.A.M de Laat, J.L.Vinke and J.J.Heijnen (2001), Energetics of growth and penicillin production in a high producing strain of Penicillium chrysogenum. Biotechnology and Bioengineering, 72(2): 185-193.

Varshney, J.L.(1981), Utilization of different carbon sources by Aspergillus clavatus and some of its mutants. Geobios. 8(4): 156-159.
Xu, X.M. (1999), Effect of temperature on the latent period of rose powdery mildew pathogen, Sphaerotheca pannosa. Plant Pathology, 48(5): 662667.

Received: June 27, 2006; Revised: April 24, 2007; Accepted: June 23, 2008 